\title{
The Role of Microaspiration in the Pathogenesis of Gastroesophageal Reflux-related Chronic Cough
}

\author{
Pelin Özdemir, ${ }^{1,2 *}$ Münevver Erdinç, ${ }^{2,3}$ Rukiye Vardar, ${ }^{2,4}$ Ali Veral, ${ }^{5}$ Serdar Akyıldız, ${ }^{2,6}$ Özer Özdemir, and Serhat Bor ${ }^{2,4}$ \\ ${ }^{I}$ Department of Chest Diseases, Su Hospital, Izmir, Turkey; ${ }^{2}$ Ege Reflux Study Group, Ege University School of Medicine, Izmir, Turkey; \\ Departments of ${ }^{3}$ Chest Diseases, ${ }^{4}$ Gastroenterology, ${ }^{5}$ Pathology, and ${ }^{6}$ Otolaryngology, Ege University School of Medicine, Izmir, Turkey; and \\ ${ }^{7}$ Department of Chest Diseases, Egepol Hospital, İzmir, Turkey
}

\section{Background/Aims}

Gastroesophageal reflux disease (GERD) is one of the main causes of chronic cough. We evaluated the role of microaspiration in the pathogenesis of reflux-related cough by determining the amount of lipid-laden macrophages (LLMs) in bronchoalveolar lavage (BAL) specimens.

\section{Methods}

A total of 161 cases of chronic cough were evaluated, and 36 patients (average age 48.2 years) were recruited for this single center prospective study. Patients with a history of smoking, angiotensin converting enzyme inhibitor usage, any abnormality on pulmonary function tests, abnormal chest X-rays, occupational or environmental exposures, or upper airway cough syndrome were excluded. GERD was evaluated by 24-hour esophageal impedance-pH monitoring. BAL specimens for LLM determination were obtained from 34 patients by flexible bronchoscopy.

\section{Results}

Patients with pathological intra-esophageal reflux according to multichannel intraluminal impedance and $\mathrm{pH}$ monitoring had higher LLM positivity in BAL specimens than patients without pathological reflux (8/14 in reflux positive group vs 1/22 in reflux negative group; $P=0.004)$. The BAL cell distribution was not different between the 2 groups $(P=0.574$ for macrophages, $P=0.348$ for lymphocytes, $P=0.873$ for neutrophils and $P=0.450$ for eosinophils).

\section{Conclusions}

Our results confirm the role of the microaspiration of refluxate in the pathogenetic mechanism of chronic cough. While bronchoscopy is indicated in patients with chronic cough, in addition to the routine airway evaluation, BAL and LLM detection should be performed. LLM can be used to diagnose aspiration in reflux-related chronic cough. Future studies are needed to evaluate the response to antireflux medications or surgery in patients with LLM positivity.

(J Neurogastroenterol Motil 2017;23:41-48)

Key Words

Cough; Gastroesophageal reflux; Macrophages, alveolar

Received: April 6, 2016 Revised: June 24, 2016 Accepted: August 15, 2016

(.) This is an Open Access article distributed under the terms of the Creative Commons Attribution Non-Commercial License (http://creativecommons. org/licenses/by-nc/4.0) which permits unrestricted non-commercial use, distribution, and reproduction in any medium, provided the original work is properly cited.

*Correspondence: Pelin Özdemir, MD

Department of Chest Diseases, Su Hospital, Güneşli District, 526/4 Str. No:7 Bulvar Apt. No:19 Konak, IZMiR 35270, Turkey Tel: +90-505-494-8336, Fax: +90-232-262-8270,E-mail: kosker_pelin@yahoo.com 


\section{Introduction}

Chronic cough is a common medical complaint. The most common causes of chronic cough (cough lasting over 8 weeks) in non-smoking adults with a normal chest radiograph include upper airway cough syndrome, gastroesophageal reflux disease, asthma and non-asthmatic eosinophilic bronchitis.

Gastroesophageal reflux disease (GERD) is one of the most common diseases in modern populations. An increased prevalence of GERD is associated with numerous factors, including lifestyle changes, such as nutritional habits, and obesity. ${ }^{1}$ The prevalence of the disease in Turkey is similar to that in Western countries, and 3 studies in Turkey showed similar prevalences ranging from $19.3 \%$ to $23 \%$. $^{2-4}$

GERD is considered to be one of the 3 main causes of chronic cough following the exclusion of parenchymal lung diseases, tobacco usage, environmental exposures and angiotensin converting enzyme inhibitor (ACEI) side effects. ${ }^{5}$ The association between GERD and chronic cough was initially based on observational studies and small treatment trials and is supported by epidemiological data. ${ }^{6,7}$ However, acid suppression with proton pump inhibitors failed to show superiority over placebo for the relief of reflux-related cough. ${ }^{8,9}$ Whether a causal relationship between these 2 conditions exists or their simultaneous occurrence is coincidental remains controversial.

Multiple mechanisms have been claimed to play a role in GERD-related cough, and cough may be provoked by the effect of refluxate on the esophagus, larynx or bronchi. There are 2 main mechanisms in the pathogenesis of reflux-related cough. ${ }^{10}$ One is named the "esophagobronchial reflex," and it is based on shared vagal innervations of the distal esophagus and airways. ${ }^{11}$ According to this theory, distal esophageal sensory nerve terminals stimulated by refluxate activate a reflex arc resulting in cough. The other mechanism is microaspiration, in which refluxate reaches the airways through the proximal esophagus and larynx directly resulting in cough. The risk of microaspiration is thought to be increased by decreased sensitivity of the protective laryngeal and pharyngeal reflexes, impaired coordination of swallowing and esophageal dysmotility. ${ }^{12}$

Different techniques, such as simultaneous measurement of esophageal and tracheal $\mathrm{pH}$, scintigraphic scans of the lungs, detection of pepsin in BAL or saliva, and detection of bile acids and lipid-laden macrophages (LLMs) in airway specimens, have been used to identify microaspiration of refluxate into airways. ${ }^{13-15}$ Stud- ies on those measurement techniques have mostly been conducted in paediatric populations. Studies in adult populations focusing on reflux, bronchoalveolar lavage (BAL) and LLMs are limited. Clinically it would be highly advantageous to have a diagnostic test that could discriminate between respiratory symptoms and diseases caused by GERD from those with other causes.

The primary aim of our study was to evaluate the role of microaspiration in patients with chronic cough due to gastroesophageal reflux disease diagnosed by multichannel intraluminal impedance and $\mathrm{pH}$ (MII-pH) monitoring. Microaspiration was indicated by the presence of LLMs in BAL specimens. The secondary aim of this study was to assess the bronchoalveolar cell distribution in patients with gastroesophageal reflux-related chronic cough.

\section{Materials and Methods}

\section{Study Population}

Patients admitted to the out-patient department of Ege University Medical Faculty Chest Disease between January 2010 and May 2011 complaining of cough lasting for 8 weeks or more were enrolled and evaluated by pulmonary and ear-nose-throat (ENT) specialists. Patients with concomitant symptoms of GERD (once a week or common heartburn and/or acid regurgitation) and pathologic reflux detected by 24-hour MII-pH monitoring were included in our study. All patients were older than 18 years of age. Written informed consent was obtained from all patients. This study was approved by the Ege University Research Ethics Committee.

The exclusion criteria were as follows: history of smoking, use of angiotensin converting enzyme inhibitors, abnormal chest $\mathrm{X}$ rays, presence of ENT pathologies, abnormal pulmonary function tests with reversibility and bronchial provocation tests, occupational or environmental exposures that could lead to pulmonary problems, immunosuppression, any treatment that can cause lipid aspiration, any other disease that can lead to aspiration, and findings compatible with upper airway cough syndrome. None of the included patients had a lipid storage disease or had ever received nutritional supplementation with lipid emulsions.

A total of 161 consecutive chronic cough patients were prospectively evaluated for this study: 36 patients were included, and 125 patients were excluded for the following reasons (Figure): 65 patients due to abnormal chest $\mathrm{X}$-rays, 32 patients due to abnormal pulmonary function test with reversibility or bronchoprovocation test, 24 patients due to findings compatible with upper airway cough syndrome, 3 patients due to ACEI usage and 1 patient due 
Assessed for eligibility $(\mathrm{N}=161)$

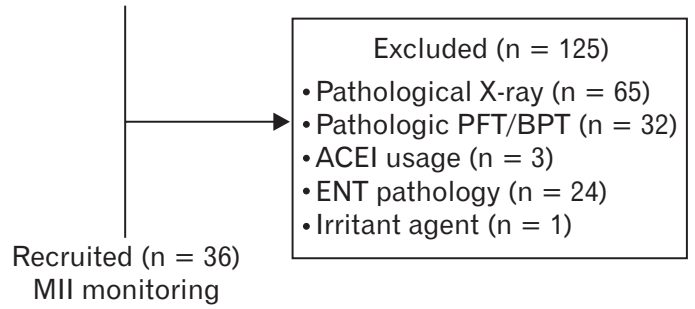

Figure. Diagram of patient recruitment. PFT, pulmonary function test; BPT, bronchoprovocation test; ACEI, angiotensin converting enzyme inhibitor; ENT, ear-nose-throat; MII, multichannel intraluminal impedance.

to environmental exposure.

\section{Pulmonary Function Tests}

Spirometry (Sensormedics 2400; Sensormedics, Yorba Linda, CA, USA) was applied to all patients. At least 3 reproducible values were obtained, and the highest value was accepted. Patients with a $\mathrm{FEV}_{1}$ value above $80 \%$ and $\mathrm{FEV}_{1} / \mathrm{FVC}$ value above $75 \%$ were considered to have normal pulmonary function. A bronchoprovocation test with methacholine was performed if the spirometric measures were normal and was evaluated using the Cockroft concentration method with a threshold of positivity of a $\mathrm{PC}_{20}$ of $16 \mathrm{mg} / \mathrm{mL}$.

\section{Combined Multichannel Intraluminal Impedance and $\mathrm{pH}$ Monitoring}

All patients were examined for pathological reflux by 24-hour MII-pH monitoring. Patients were asked to stop proton pump inhibitors and $\mathrm{H}_{2}$ receptor blocking drugs for 7 days and to stop drugs affecting the lower esophageal sphincter for 48 hours prior to the procedure. After detection of lower esophageal sphincter localization by high-resolution manometric examination, a MII catheter (2-4-6-8-10-14-16th cm impedance rings and 5th $\mathrm{cm} \mathrm{pH}$ electrode) was placed to provide a $\mathrm{pH}$ probe at $5 \mathrm{~cm}$ above the LES transnasally that was connected to a portable data recorder (Ohmega; MMS, Enschede, The Netherlands). Twenty-four-hour monitoring was carried out, and the patients were instructed to have a usual day with a regular diet and to push the symptom button whenever they had symptoms of reflux. pH changes were recorded, and the data were analysed after the test was completed. Only liquid refluxes, defined as a retrograde drop in impedance of $50 \%$ or more from the baseline, were evaluated. A pH of $<4$ was defined as acidic reflux, whereas a $\mathrm{pH}$ of 4-7 was defined as weak acid reflux and a $\mathrm{pH}$ of $>7$ was defined as nonacidic reflux. Normal values of total distal reflux were total reflux events $\leq 73$, acid reflux events $\leq$
55 , weakly acid reflux events $\leq 26$ and weakly alkaline reflux $\leq 1$ based on the 95 th percentile as the upper limit of the normal. Normal values for impedance $\mathrm{pH}$ monitoring were $\%$ bolus exposure (impedance parameter) and $\%$ acid exposure ( $\mathrm{pH}$ parameter): total $1.4 \%$; $6.3 \%$; upright: $2.1 \%, 9.7 \%$; and recumbent: $0.7 \%, 2.1 \% .^{16,17}$

\section{Bronchoscopy and Bronchoalveolar Lavage}

Except for 4 patients who declined to give informed consent, transnasal or transoral bronchoscopic examination was performed in all patients with a fiberoptic bronchoscope (Olympus BF-2T10; Olympus Co, Tokyo, Japan). Local anaesthesia (2\% lidocaine $\mathrm{HCl}$, topical, maximum $10 \mathrm{~mL}$ ) and premedication with midazolam were applied before the procedure. The bronchial branches on both sides were assessed for macroscopic abnormality. The middle lobe medial segment bronchus was wedged, and 120-150 mL saline was instilled for BAL. The lavage material was filtered before cytological examination.

BAL specimens were examined by liquid-based cytology and slides prepared on a Thinprep device were stained with Sudan Black to detect LLM positivity. The cells with cytoplasmic orangered granules was counted as positive. The pathologist who evaluated the BAL specimens was blinded to the patients' GERD status.

\section{Examination for Upper Airway Cough Syndrome}

All patients underwent an examination by an ENT specialist before the reflux work-up. A reflux finding score was obtained to evaluate laryngopharyngeal reflux, as described in the literature. ${ }^{18} \mathrm{~A}$ score greater than 7 was considered positive for laryngopharyngeal reflux, whereas a score greater than 11 was considered strongly suggestive of laryngopharyngeal reflux.

\section{Statistical Methods}

Statistical analyses were performed using SPSS v16 (SPSS for Windows; SPSS Inc, Chicago, IL, USA). Fisher's exact test and $\chi^{2}$ test were used to compare the MII-pH monitoring data and BAL LLM positivity. Reflux index, impedance $\mathrm{pH}$-metry parameters and BAL cell distribution were evaluated by nonparametric statistical analysis, as data were not normally distributed. The Mann Whitney $U$ test was used to compare the reflux positive group to the control group. $P$ levels below 0.05 were considered statistically significant.

\section{Results}

A total of 161 patients were screened, and 36 patients were 
included in this study; $78 \%$ of the included patients were female, and the mean age of the patients was $48.2 \pm 12.9$ years. The average cough time of the patients was $35.1 \pm 35.6$ months (3-180 months). Thirty-four patients had never smoked, one patient had a smoking history of 3 pack-years and one had a smoking history of 4 pack-years. All patients included in this study had normal pulmonary function test values and normal bronchoprovocation test results with methacholine.

Fourteen patients $(38.9 \%)$ had pathological reflux detected by MII-pH monitoring (pathological reflux [+] group). The control group was composed of 22 patients $(61.1 \%)$ whose MII-pH monitoring results were considered normal (pathological reflux [-] group). In the pathological reflux group, the average incidences of different levels of reflux were as follows: total reflux, $90.4 \pm 38.7$; acidic reflux, $56.4 \pm 27.4$; weak acidic reflux, $33.7 \pm 23.3$; and nonacidic reflux, $0.2 \pm 0.6$.

A total of $25.0 \%(\mathrm{n}=9)$ of all patients had BAL LLM positivity. While 8 out of 14 patients $(57.1 \%)$ in the pathological reflux group had LLM positivity, only one patient (4.5\%) in the control group had LLM positivity. Compared with the control group, patients with pathological reflux had significantly higher BAL LLM positivity $(P=0.004)$.

Reflux finding scores, which were evaluated by an ENT specialist, were significantly higher in the pathological reflux group: in the pathological reflux group median was 14 with an interquartile range (IQR) of 4; whereas in the control group median was 6 with an IQR of $8(P<0.001)$. The comparison of the pathological reflux group and control group is presented in Table 1.

When LLM-positive and LLM-negative groups were compared, the reflux finding scores were significantly higher in the LLM-positive group (median 14, IQR 4 vs median 6, IQR 8;
$P<0.001)$. The incidence of total reflux and the acidic reflux numbers were higher in the LLM-positive group than the LLMnegative group (respectively, for total reflux median 76, IQR 57 vs median 43, IQR 32; $P=0.032$ and for acidic reflux median 53.5, IQR 49 vs median 18 , IQR 57; $P=0.003$ ). The comparison of LLM-positive and LLM-negative groups is shown in Table 2. LLM positivity according to GERD status is shown in Table 3.

Considering all patients, on average, the BAL cell distribution was $87.9 \%$ for macrophages, $8.8 \%$ for lymphocytes, and $3 \%$ for neutrophils. There were no significant differences between the pathological reflux group and the control group in regard to BAL macrophage, lymphocyte, neutrophil or eosinophil distributions ( $P=0.574,0.348,0.873$, and 0.450 , respectively). Similarly, there were no significant differences in BAL macrophage, lymphocyte, neutrophil or eosinophil distributions between the LLM-positive and LLM-negative groups ( $P=0.472,0.443,0.743$, and 0.775 , respectively).

\section{Discussion}

Main mechanisms of reflux associated chronic cough are the esophago-bronchial vagal reflex mechanism and microaspiration. In this study we investigated the role of microaspiration in patients with gastroesophageal reflux-associated chronic cough by measuring LLM positivity in BAL specimens. Patients with pathological reflux according to $\mathrm{MII} / \mathrm{pH}$ monitoring findings had significantly higher BAL LLM positivity compared to patients without pathological reflux, suggesting the importance of microaspiration. On the other hand, patients with LLM positivity had a higher incidence of total and acidic reflux compared to patients who were negative for LLM. BAL cell distributions were not different between the

Table 1. Comparison of Pathological Reflux Group and Control Group

\begin{tabular}{|c|c|c|c|}
\hline & $\begin{array}{l}\text { Pathological reflux }(+) \\
\qquad(\mathrm{n}=14)\end{array}$ & $\begin{array}{l}\text { Pathological reflux }(-) \\
\qquad(\mathrm{n}=22)\end{array}$ & $P$-value \\
\hline Age (median $\pm \mathrm{SD}$, yr) & $46.1 \pm 12.5$ & $49.5 \pm 13.2$ & 0.445 \\
\hline Duration of cough (median $\pm \mathrm{SD}, \mathrm{mo})$ & $34.1 \pm 16.6$ & $35.8 \pm 44.0$ & 0.876 \\
\hline LLM (n [\%]) & $8(57.1)$ & $1(4.5)$ & 0.004 \\
\hline \multicolumn{4}{|l|}{ BAL cell distribution (median $[\mathrm{IQR}]$ ) } \\
\hline Macrophages & $91.5(7)$ & $90.0(6)$ & 0.574 \\
\hline Lymphocytes & $6.0(4)$ & $8.0(4)$ & 0.348 \\
\hline Neutrophils & $3.0(4)$ & $3.0(3)$ & 0.873 \\
\hline Eosinophils & $0.0(1)$ & $0.0(1)$ & 0.450 \\
\hline Reflux finding score & $14.0(4)$ & $6.0(8)$ & $<0.001$ \\
\hline
\end{tabular}

LLM, lipid-laden macrophage; BAL, bronchoalveolar lavage; IQR, interquartile range. 
Table 2. Comparison of Lipid-laden Macrophage Positive and Lipid-laden Macrophage Negative Patients

\begin{tabular}{lccr}
\hline & LLM $(+)$ & LLM $(-)$ \\
$(\mathrm{n}=27)$ & $P$-value \\
& $(\mathrm{n}=9)$ & $46.7 \pm 13.2$ & 0.646 \\
Age (median $\pm \mathrm{SD}, \mathrm{yr})$ & $49.0 \pm 12.5$ & $35.0 \pm 42.3$ & 0.842 \\
Duration of cough (median $\pm \mathrm{SD}, \mathrm{mo})$ & $38.1 \pm 16.7$ & $6.0(8)$ & 0.001 \\
Reflux finding score (median [IQR]) & $14.0(4)$ & $43.0(32)$ & 0.032 \\
Total reflux number (median [IQR]) & $76.0(57)$ & $18.0(18)$ & 0.003 \\
Acidic reflux (median [IQR]) & $53.5(49)$ & $26.0(19)$ & 0.968 \\
Weak acidic reflux (median [IQR]) & $20.2(25)$ & $1.0(4)$ & 0.030 \\
Nonacidic reflux (median [IQR]) & $0.0(0)$ & $88.0(6)$ & 0.472 \\
BAL cell distribution (median [IQR]) & $92.0(6)$ & $8.0(3)$ & 0.443 \\
Macrophages & $5.5(5)$ & $3.0(4)$ & 0.743 \\
Lymphocytes & $2.5(2)$ & $0.0(1)$ & 0.775 \\
Neutrophils & $0.0(1)$ & \\
Eosinophils & & \\
\hline
\end{tabular}

LLM, lipid-laden macrophages; IQR, interquartile range; BAL, bronchoalveolar lavage.

Table 3. Lipid-laden Macrophage Positivity According to Gastroesophageal Reflux Disease Status

\begin{tabular}{ccc} 
& GERD $(+)$ & GERD $(-)$ \\
\hline $\operatorname{LLM}(+)$ & 8 & 1 \\
$\operatorname{LLM}(-)$ & 6 & 21 \\
\hline
\end{tabular}

GERD, gastroesophageal reflux disease; LLM, lipid-laden macrophages. Data are presented as number of patients.

groups. The reflux finding scores at the pharynx and larynx scored by an ENT specialist supported the BAL findings, with a significantly high score in the pathological reflux group compared to that in the reflux-negative group. The difference in the reflux finding scores between the LLM-positive and LLM-negative patients was also statistically significant $(P<0.001)$.

Early studies have mostly focused on the shared vagal innervations of the esophagus and airways as the main mechanism of chronic cough because cough episodes were temporally associated with distal esophageal acidification in patients with normal chest Xray, bronchoscopy or laryngeal examination. ${ }^{19}$ Additionally, refluxrelated cough was suppressed with esophageal lidocaine instillation or tracheal ipratropium inhalation. ${ }^{11}$ Javorkova et $\mathrm{al}^{20}$ showed that the acidification of the distal esophagus increased cough sensitivity in patients with GERD and chronic cough in comparison to healthy volunteers and patients with GERD only. This effect was also observed in asthma patients without cough or GERD. ${ }^{21}$ These findings support the concept that this reflex may not be the sole mechanism of reflux cough and that some airway disease may be needed for cough sensitivity to occur. Although in chronic cough patients, proximal esophageal reflux episodes do not seem to be dif- ferent than in healthy volunteers, ${ }^{22}$ microaspiration of gastric content into the airways is considered to be one of the causes of cough. Dysfunction of the upper esophageal sphincter and insufficiency of protective laryngeal and pharyngeal reflexes may predispose patients to microaspiration. ${ }^{23}$ Simultaneous esophageal and tracheal $\mathrm{pH}$ drops during reflux episodes in asthma patients supports this hypothesis. ${ }^{13}$

The presence of pepsin, bile acids or LLMs in BAL specimens has been proposed as an index to establish the degree of gastric aspiration. The LLM index is mostly used to detect aspiration-induced chronic lung diseases in pediatric patients, especially in cases that are due to chronic aspiration. ${ }^{24}$ The value of that index has also been confirmed in an adult patient population with aspiration pneumonia. ${ }^{25}$ The importance of LLM positivity in assessing GERD was shown in cystic fibrosis patients with lung transplantation by Hayes et al. ${ }^{26}$ In that study, some patients underwent laparoscopic Nissen fundoplication and others underwent anti-reflux medical therapy. The LLM index was significantly lower in the surgical group. Opposing the reliability of LLM index as a marker of microaspiration, Kitz et $\mathrm{al}^{27}$ conducted a study in children with GERD related respiratory symptoms and found no association between reflux events and LLM counts. But MII was not used in this study, and also underlying pulmonary disease of the selected patient population may cause more reduction in sensitivity and specificity of LLM index.

However, LLM presence in BAL may not differentiate direct aspiration to the airways from secondary aspiration of reflux. Additionally, lipids in macrophages may include endogenous lipids as well as exogenous lipids, as observed in reflux material. Any disorder leading to pulmonary inflammation may cause LLM positivity. 
In our study, the patient population was carefully chosen to avoid these problems by excluding patients with any airway or lung disease, radiographic abnormality or any environmental exposure that can cause airway inflammation. Patients with bronchial obstruction, malignancy, and organic dust exposure were also excluded, as those conditions may result in endogenous lipid phagocytosis of macrophages. It must be kept in mind that in the opinion of some researchers, LLM presence in BAL may not be an adequate test for aspiration because of the absence of reference values in healthy volunteers and the probable discrepancy between observers, as it is scored visually.

In our study 6 out of 14 patients with GERD showed LLM negativity, and 1 control patient showed LLM positivity. Regarding the sensitivity and specificity of LLM positivity, there may be false negative or false positive results. Maybe these are the results of other causes of LLM positivity that are mentioned above. Also it should be kept in mind that there is no gold standard test for GERD diagnosis. Other markers, like pepsin and bile acids are also used as markers of microaspiration. In a study by Stovold et $\mathrm{al}^{28} \mathrm{BAL}$ pepsin levels were found to be elevated in lung transplant recipients, with the highest levels in subjects with acute vascular rejection. BAL pepsin levels of GERD patients with and without chronic cough were found to be similar and lower. In a study of Grabowski et $\mathrm{al}^{29}$ pepsin and bile acids in induced sputum of chronic cough patients were not different between GERD patients and the control group. In a study of Decalmer et al, ${ }^{30}$ BAL pepsin levels were found to be lower in chronic cough patients compared with controls, and they found no correlation between proximal and distal reflux events and BAL pepsin concentration. The results of these studies conflicts with our findings. This confliction may have some several probable methodological reasons. In the study of Grabowski et $\mathrm{al}^{29}$ GERD diagnosis was based on gastroscopy findings and treatment response, multichannel impedance, and $\mathrm{pH}$-metry was not used and also pepsin levels were detected in sputum samples, not in BAL. In the study of Decalmer et al, ${ }^{30}$ selection of the study population was different, mainly considering unexplained chronic cough patients with various respiratory diagnoses or GERD including ex-smokers. It should also be kept in mind that pepsin measurement is also not validated as a marker of aspiration because of some significant methodological issues, like cross reaction of the immunologic assay with pepsinogen. ${ }^{31}$ For aspiration to occur, the refluxate should traverse the length of the esophagus and reach the larynx and pharynx. Although pharyngeal reflux is not prominent in chronic cough patients, our findings indicate that it may occur more often in these patients. ${ }^{32}$ Because of the technical difficulties of placement of 2 catheters or placement of an impedance catheter with $2 \mathrm{pH}$ probes, proximal reflux events were not evaluated in our study. However, the reflux finding score was positive in 20 (55.6\%) patients, although the accuracy of evaluating proximal esophageal reflux with this score is arguable, as it depends on a subjective visual scoring system. Limited data exist about the value of proximal $\mathrm{pH}$ probe monitoring in patients with chronic cough and GERD; however, many studies have shown the inefficiency of this technique for the diagnosis of laryngopharyngeal reflux.$^{33}$

MII-pH monitoring for the diagnosis of GERD allowed for the detection of gaseous and weak-nonacidic reflux in addition to acidic and liquid reflux. Proximal reflux episodes and nonacidic reflux associated with cough episodes have been observed in a small portion of the patient population, and an increase in reflux episodes in patients with chronic cough compared to healthy volunteers is seldom observed. This finding indicates the possibility that even physiological reflux can provoke cough in these patients.

There was no significant difference in the BAL cell distribution between the pathological reflux group and control group or between the LLM $(+)$ and LLM (-) groups. In a study by Chang et $\mathrm{al},{ }^{34}$ GERD patients without any pulmonary disease showed no difference in BAL cell distribution or LLM positivity compared with controls. According to these limited data, BAL cell distribution changes may be attributed to pulmonary diseases, not GERD itself. This study of Chang et al, ${ }^{34}$ differs from our results regarding LLM positivity. This difference may be due to difference of selected populations, as children with gastrointestinal symptoms with indication of gastroendoscopy was included in that study. Also GERD diagnosis methods and BAL were different.

Our study has some limitations. We tried to recruit patients with isolated true reflux cough: patients with any sign of airway or parenchymal disease, upper airway cough syndrome, or any other condition that can result in cough were excluded. This choice caused the patient population to be small, although it was one of the largest groups used to study this topic in the literature. Acidic and nonacidic reflux episodes were identified by $\mathrm{MII} / \mathrm{pH}$ monitoring, but proximal reflux episodes were not evaluated with a proximal $\mathrm{pH}$ probe.

As a result, based on the hypothesis that esophagobronchial reflex and microaspiration are the 2 main mechanisms of reflux cough, our results confirm the role of refluxate microaspiration in the pathogenetic mechanism of chronic cough. In patients with chronic cough, who represents a challenging patient group in pulmonary medicine, LLM in BAL specimens may indicate the presence of microaspiration due to GERD; however, larger 
controlled trials are necessary to determine the reference values for this evaluation. It should be taken into consideration that although bronchoscopy is indicated in patients with chronic cough, routine airway evaluation, BAL and LLM staining should also be performed. Future studies are needed to evaluate the response to antireflux medications and surgery in patients with LLM positivity.

\section{Financial support: None.}

\section{Conflicts of interest: None.}

Author contributions: Pelin Özdemir, Rukiye Vardar, Münevver Erdinç, and Serhat Bor planned and designed the study; Pelin Özdemir and Rukiye Vardar took role in recruitment, enrolment, and assessment of participants; Pelin Özdemir and Özer Özdemir wrote the manuscript; Pelin Özdemir, Rukiye Vardar, Serhat Bor, Münevver Erdinç, and Serdar Akyıldız analysed and interpreted data; Münevver Erdinç and Serhat Bor supervised the study; Ali Veral performed pathological preparations and evaluation; and Serdar Akyldız performed otolaryngological examination. All authors approved the final draft.

\section{References}

1. Bonatti $\mathrm{H}$, Achem SR, Hinder RA. Impact of changing epidemiology of gastroesophageal reflux disease on its diagnosis and treatment. J Gastrointest Surg 2008;12:373-381.

2. Bor S, Mandiracioglu A, Kitapcioglu G, Caymaz-Bor C, Gilbert RJ. Gastroesophageal reflux disease in a low-income region in Turkey. Am J Gastroenterol 2005;100:759-765.

3. Bor S, Kitapcioglu G, Kasap E, Ilter, T. The prevalence of gastroesophageal reflux disease in Turkey: lessons from a high Helicobacter pylori prevalent country. J Clin Gastroenterol 2006;40:S182-S183.

4. Mungan Z. Prevalence and demographic determinants of gastroesophageal reflux disease (GERD) in the Turkish general population. population: a population-based cross-sectional study. Turk J Gastroenterol 2012;23:323-332.

5. Palombini BC, Villanova CA, Araújo E, et al. A pathogenic triad in chronic cough: asthma, postnasal drip syndrome, and gastroesophageal reflux disease. Chest 1999;116:279-284.

6. Irwin RS, Curley FJ, French CL. Chronic cough: the spectrum and frequency of causes, key components of the diagnostic evaluation, and outcome of specific therapy. Am Rev Respir Dis 1990;141:640-647.

7. Ing AJ, Ngu MC, Breslin AB. Pathogenesis of chronic persistent cough associated with gastroesophageal reflux. Am J Respir Crit Care Med 1994;149:160-167.

8. Shaheen NJ, Crockett SD, Bright SD, et al. Randomised clinical trial: high-dose acid suppression for chronic cough - a double-blind, placebo- controlled study. Aliment Pharmachol Ther 2011:33:225-234.

9. Faruqi S, Molyneux ID, Fathi D, Wright C, Thompson R, Morice AH. Chronic cough and esomeprazole: a double-blind placebo-controlled parallel study. Respirology 2011;16:1150-1156.

10. Smith JA, Abdulqawi R, Houghton LA. GERD-related cough: pathophysiology and diagnostic approach. Curr Gastroenterol Rep 2011;13:247-256.

11. Ing AJ, Ngu MC, Breslin ABX. Chronic persistent cough and gastroesophageal reflux. Thorax 1991;46:479-483.

12. Phua SY, McGarvey LP, Ngu MC, Ing AJ. Patients with gastrooesophageal reflux disease and cough have impaired laryngopharyngeal mechanosensitivity. Thorax 2005;60:488-491.

13. Jack CI, Calverley PM, Donnelly RJ, et al. Simultaneous tracheal and oesophageal $\mathrm{pH}$ measurements in asthmatic patients with gastro-oesophageal reflux. Thorax 1995;50:201-204.

14. Stein MR. Possible mechanisms of influence of esophageal acid on airway hyperresponsiveness. Am J Med 2003;115(suppl 3A):55S-59S.

15. Grabowski M, Kasran A, Seys S, et al. Pepsin and bile acids in induced sputum of chronic cough patients. Respir Med 2011;105:1257-1261.

16. Shay S, Tutuian R, Sifrim D, et al. Twenty-four hour ambulatory simultaneous impedance and $\mathrm{pH}$ monitoring: a multicenter report of normal values from 60 healthy volunteers. Am J Gastroenterol 2004;99:10371043.

17. Zentilin P, Iiritano E, Dulbecco P, et al. Normal values of 24-h ambulatory intraluminal impedance combined with $\mathrm{pH}$-metry in subjects eating a Mediterranean diet. Dig Liver Dis 2006;38:226-232.

18. Belafsky PC, Postma GN, Koufman JA. The validity and reliability of the reflux finding score (RFS). Laryngoscope 2001;111:1313-1317.

19. Irwin RS, French CL, Curley FJ, Zawacki JK, Bennett FM. Chronic cough due to gastroesophageal reflux. Clinical, diagnostic, and pathogenetic aspects. Chest 1993;104:1511-1517.

20. Javorkova N, Varechova S, Pecova R, et al. Acidification of the oesophagus acutely increases the cough sensitivity in patients with gastro-oesophageal reflux and chronic cough. Neurogastroenterol Motil 2008;20:119124

21. Wu DN, Yamauchi K, Kobayashi H, et al. Effects of esophageal acid perfusion on cough responsiveness in patients with bronchial asthma. Chest 2002;122:505-509.

22. Blondeau K, Dupont LJ, Mertens V, Tack J, Sifrim D. Improved diagnosis of gastro-oesophageal reflux in patients with unexplained chronic cough. Aliment Pharmacol Ther 2007;25:723-732.

23. Smith JA, Houghton LA. The oesophagus and cough: laryngo-pharyngeal reflux, microaspiration and vagal reflexes. Cough 2013;9:12.

24. Corwin RW, Irwin RS. The lipid-laden alveolar macrophage as a marker of aspiration in parenchymal lung disease. Am Rev Respir Dis 1985;132:576-581.

25. Adams R, Ruffin R, Campbell D. The value of the lipid-laden macrophage index in the assessment of aspiration pneumonia. Aust $\mathrm{N} Z \mathrm{~J}$ Med 1997;27:550-553.

26. Hayes D Jr, Kirkby S, McCoy KS, et al. Reduction of lipid-laden macrophage index after laparoscopic Nissen fundoplication in cystic fibrosis patients after lung transplantation. Clin Transplant 2013;27:121-125. 
27. Kitz R, Boehles HJ, Rosewich M, Rose MA. Lipid-laden alveolar macrophages and $\mathrm{pH}$ monitoring in gastroesophageal reflux-related respiratory symptoms. Pulm Med 2012;2012:673637.

28. Stovold R, Forrest IA, Corris PA, et al. Pepsin, a biomarker of gastric aspiration in lung allografts: a putative association with rejection. Am J Respir Crit Care Med 2007;175:1298-1303.

29. Grabowski M, Kasran A, Seys S, et al. Pepsin and bile acids in induced sputum of chronic cough patients. Respir Med 2011;105:1257-1261.

30. Decalmer S, Stovold R, Houghton LA, et al. Chronic cough: relationship between microaspiration, gastroesophageal reflux, and cough frequency. Chest 2012;142:958-964.

31. Trinick R, Johnston N, Dalzell AM, McNamara PS. Reflux aspiration in children with neurodisability--a significant problem, but can we measure it? J Pediatr Surg 2012;47:291-298.

32. Sifrim D, Dupont L, Blondeau K, Zhang X, Tack J, Janssens J. Weakly acidic reflux in patients with chronic unexplained cough during 24 hour pressure, $\mathrm{pH}$, and impedance monitoring. Gut 2005;54:449-454.

33. Bilgen C, Ogüt F, Kesimli-Dinç H, Kirazli T, Bor S. The comparison of an empiric proton pump inhibitor trial vs. 24-hour double-probe $\mathrm{pH}$ monitoring in laryngopharyngeal reflux. J Laryngol Otol 2003;117:386390.

34. Chang AB, Cox NC, Purcell J, et al. Airway cellularity, lipid laden macrophages and microbiology of gastric juice and airways in children with reflux oesophagitis. Respir Res 2005;6:72. 